\title{
Féeries
}

Études sur le conte merveilleux, XVII ${ }^{\mathrm{e}} \mathrm{XIX} \mathrm{X}^{\mathrm{e}}$ siècle

Le Conte oriental

\section{Les notes dans les premiers contes orientaux}

\section{Christelle Bahier-Porte}

\section{(2) OpenEdition}

\section{Journals}

Édition électronique

URL : http://journals.openedition.org/feeries/106

DOI : $10.4000 /$ feeries. 106

ISSN : 1957-7753

\section{Éditeur}

UGA Éditions/Université Grenoble Alpes

\section{Édition imprimée}

Date de publication : 1 février 2005

Pagination : 91-108

ISBN : 2-84310062-3

ISSN : $1766-2842$

Référence électronique

Christelle Bahier-Porte, «Les notes dans les premiers contes orientaux », Féeries [En ligne], 2 | 2005, mis en ligne le 29 mars 2007, consulté le 10 décembre 2020. URL : http://journals.openedition.org/ feeries/106; DOI : https://doi.org/10.4000/feeries.106

Ce document a été généré automatiquement le 10 décembre 2020.

(C) Féeries 


\title{
Les notes dans les premiers contes orientaux
}

\author{
Christelle Bahier-Porte
}

1 LA VOGUE DU CONTE ORIENTAL naît avec la parution du premier tome des Mille et Une Nuits traduites par Antoine Galland en 1704. Indéniablement, ces contes offrent une nouvelle matière et une nouvelle manière pour le conte, mais ils comblent aussi une curiosité déjà attisée par les relations de voyageurs et les visites d'ambassadeurs orientaux en France. Le conte oriental se définirait alors par un statut hybride apte à répondre à cette curiosité du public pour le monde oriental: il fait découvrir une nouvelle civilisation, fascinante, et révèle ainsi une vocation documentaire tout en reposant sur un nouveau type de merveilleux qui comble l'imagination des lecteurs après une relative lassitude pour les contes de fées plus traditionnels. Ce qui explique alors que les premiers contes orientaux nous sont parvenus par le biais d'éminents orientalistes, Antoine Galland et François Pétis de La Croix. On présente souvent les contes de ces deux savants comme des délassements, des amusements au cœur de travaux plus " sérieux », pourtant leur spécificité, leur qualité peut-être, naît de ce savoir précis sur l'Orient allié à un talent de conteur. D'ailleurs, cette double vocation du conte oriental est affirmée dans l'Avertissement du premier tome des Mille et Une Nuits: Galland promet des contes «agréables et divertissants » grâce au merveilleux et aux nombreuses péripéties qui les composent mais ajoute : «ils doivent plaire encore par les coutumes et les mœurs des Orientaux" et suggère même que les informations seront plus précises ou plus fiables que dans les récits des voyageurs : " ces choses y sont mieux marquées que dans les auteurs qui en ont écrit et que dans les relations des voyageurs ${ }^{1} »$.

2 La présence de notes infrapaginales dans les premiers recueils de contes orientaux est alors un signe ostensible de cette double vocation du conte oriental, au point qu'elle est stigmatisée comme stéréotype par Crébillon dans la préface de Tanzaï et Néadarné ${ }^{2}$. Les notes sont peu nombreuses encore dans Les Mille et Une Nuits, elles prennent une place plus significative dans Les Mille et Un Jours. L'étude de la nature et de la fonction de ces notes nous permettra de mettre en lumière, incidemment, le statut particulier du conte 
oriental à son apparition, chargé de répondre à une double attente savante et fictionnelle. Ce peut être une sorte de gageure que d'allier fiction et érudition et la note, parce qu'elle est ostensible et de lecture facultative, est peut-être précisément le signe et le stigmate de cette gageure: au cours de l'histoire du conte oriental, l'imagination finit par l'emporter sur la vocation documentaire. Après avoir rappelé l'horizon d'attente du conte oriental au début du dix-huitième siècle dont la curiosité est le maître mot, nous étudierons le système des notes dans Les Mille et Un Jours, caractéristique de l'ambivalence de la «note de conte» entre érudition et distanciation. Gueullette choisit, quant à lui, de se faire un jeu de l'érudition et montre, dès 1715 , que la note apparaît comme un élément obligé du conte oriental. Ce sera moins vrai quand l'Orient ne sera plus qu'un décor pour des fictions de plus en plus folles mais quand Caylus veut redonner quelque "sérieux» au conte oriental, il retrouve l'usage des notes.

La curiosité pour l'Orient

3 Il faut bien avouer qu'aujourd'hui encore il est difficile d'avoir une idée spontanément précise de cette vaste région ${ }^{3}$. Pour tenter de la circonscrire et donc de la dominer quelque peu, on parle à la fin du dix-neuvième siècle, de Moyen-Orient pour désigner les régions riveraines de la Méditerranée, de la Mer rouge, du golfe d'Oman et du golfe persique (la Turquie, la Syrie, l'Arabie Saoudite, le Liban, l'Égypte, l'Irak et l'Iran en font partie) et d'Extrême-Orient pour les pays d'Asie situés à l'est du détroit de Malacca (la Chine, le Japon, la Thaïlande par exemple). Au dix-huitième siècle, on est bien loin de ces nuances. Pour le grand public, l'Orient est une notion vague renvoyant davantage à des mystères et des légendes qu'à des découpages géographiques précis. L'Orient, c'est l'endroit où le soleil se lève (orior en latin) mais c'est surtout un "ailleurs". On distingue généralement l'Orient religieux: c'est l'orient des Musulmans, une civilisation établie à laquelle s'oppose celle des Chrétiens et un Orient inconnu que l'on situe vaguement aux confins de la Chine et que l'on associe aux légendes et à l'idolâtrie. L'Orient se définit donc d'abord par opposition à l'Occident et surtout par opposition à une religion. C'est pourquoi, on distingue, au sein de l'Orient religieux, un «Orient sacré », celui de la Terre Sainte autour de Jérusalem, dont l'histoire est liée à celle du christianisme. Cet Orient sacré n'est pas représenté dans les œuvres littéraires du dixhuitième siècle. On peut donc retenir, avec Mohamed Abdel-Halim ${ }^{4}$, ce premier critère de définition du monde oriental: une religion musulmane ou idolâtre. D'un point de vue strictement géographique, l'Orient regroupe de nombreux pays ou régions. Antoine Galland définit ainsi les Orientaux: "Sous le nom d'Orientaux, je ne comprends pas seulement les Arabes et les Persans mais encore les Turcs et les Tartares ${ }^{5}$ et presque tous les peuples de l'Asie jusqu'à la Chine, Mahométans ou païens et idolâtres ${ }^{6}$ ». On voit que le critère religieux est spontanément associé à l'énumération géographique. On parcourt dans Les Mille et Un Jours : l'Égypte, la Syrie, la Mésopotamie (l'Irak actuel avec Bagdad), le Turkestan (Samarcande, Cogende, Cachgar), l'Afghanistan (Gazna, Kandahar), le Pakistan (Caracorom), la Chine, la province de Cachemire (Au nord du Pakistan actuel), le Tibet, l'Inde (Surate), les îles de Sérendib (Ceylan), de Java, de Sumatra...

4 L'Orient au début du dix-huitième siècle, terre aux frontières imprécises, serait d'abord un Orient de rêve associé à des images chatoyantes et sensuelles. Etiemble rappelle néanmoins, au début de son cours sur L'Orient philosophique au dix-huitième siècle, qu'il ne faut pas croire que l'intérêt pour l'Orient a un commencement absolu au siècle des lumières : «cette influence était préparée depuis des siècles par une curiosité souvent 
en éveil et par des relations terrestres et maritimes qui furent rarement tout à fait interrompues ${ }^{7}$ ». En effet, les voyageurs, les commerçants, les missionnaires proposent, chacun à leur manière, une "image " de l'Orient et sont ainsi à l'origine d'un mouvement de curiosité qui servira la vogue du conte oriental. Les relations commerciales encouragées par Colbert à partir de 1660 grâce à la création de grandes compagnies comme la Compagnie de la Chine (1660) ou la Compagnie du Levant (1670) développent le goût exotique du public. Les négociants rapportent des épices, des bibelots, des étoffes qui provoquent l'enthousiasme.

5 La curiosité est également attisée par les récits des ambassadeurs français en Orient diffusés dans les journaux. Par exemple, on peut lire dans Le Mercure galant d'octobre 1712 la « relation de l'audience de Monsieur d'Agatopolis au Sophi de Perse » avec force détails qui ne sont pas seulement liés à la politique: "On offrit à M. d'Agatopolis quantité de confitures et ensuite plusieurs grands plats d'or garnis de viandes à la manière du pays ». Mais c'est surtout la venue des ambassadeurs orientaux en France qui captive les foules. La population française admire devant elle un représentant de ces hommes d'Orient qui fascinent tant voyageurs et commerçants, même s'il s'agit plutôt d'une « représentation ». En effet, la visite d'un ambassadeur est l'objet d'une véritable mise en scène théâtrale: costumes, cortège imposant, révérences, tout est orchestré pour frapper les imaginations. Ces ambassades ont un retentissement considérable, la plus célèbre est sans doute celle de Suleiman Aga en 1669 et 1670 qui inspira Molière ${ }^{8}$. Hélène Desmet-Grégoire, qui a étudié les différentes ambassades turques, remarque que cet enthousiasme est lié aux débuts de la diffusion de la matière d'Orient car les ambassades de 1581 et 1618 avaient eu beaucoup moins de succès. Ces visites influencent par ailleurs les représentations mentales de l'ensemble de l'Orient ${ }^{9}$. L'appréhension de l'Orient est donc d'abord empirique : les ambassadeurs en donnent une image, les commerçants en donnent une idée, les missionnaires une autre. Ces derniers sont par exemple à l'origine du cliché de la Chine savante et philosophe, image que l'on retrouvera dans les œuvres philosophiques de Voltaire ${ }^{10}$.

6 Cependant, les plus grands producteurs d'images, qui deviendront autant de topoï du conte oriental, sont probablement les voyageurs. Selon Pierre Martino, jusqu'à aux années 1660 , les voyageurs donnent "une image insuffisante ${ }^{11}$ " de l'Orient, ne retenant que ce qui ressemble à la France en particulier du point de vue des administrations et du gouvernement. De 1660 à 1750, les récits de voyage se multiplient et les voyageurs sont désormais des hommes d'autorité - Bernier était par exemple docteur en médecine -, rapportant de leurs séjours des comptes rendus précis. Outre des descriptions des pays traversés, les voyageurs s'essayent à définir l'Homme oriental. Trois grands thèmes sont particulièrement mis en relief. Le despotisme fascine les voyageurs : " Il n'y a assurément aucun souverain au monde si absolu que le roi de Perse ; car on exécute toujours exactement ce qu'il prononce sans avoir égard, ni au fond, ni aux circonstances des choses " écrit par exemple Chardin en 1672 dans Les Beautés de la Perse ${ }^{12}$; la conception matérielle du paradis comme jardin enchanteur et le fatalisme placide des Orientaux reviennent également dans bon nombre de relations. Enfin les auteurs sont inépuisables sur les sérails, les eunuques, les passions extrêmes, Bernier écrit par exemple : «Ce ne sont pas des amourettes comme les nôtres qui n'ont que des aventures galantes et comiques, elles sont toujours suivies de quelque chose d'horrible et de funeste ${ }^{13}$ ». 
7 Les voyageurs, les négociants et les missionnaires ont ainsi créé l'horizon d'attente du conte oriental, d'autant plus que certains récits de voyage peuvent être perçus comme les premiers essais littéraires sur l'Orient. Marie-Louise Dufrénoy met ainsi en avant les «dons exceptionnels de conteur » de François Bernier qui voyage en Égypte et surtout en Inde et passe huit ans à la cour du Grand Mogol. L'Histoire de la dernière Révolution des états $d u$ Grand Mogol (1670) laisse place à de nombreuses anecdotes comiques ou tragiques et Bernier «excelle à rehausser ses récits de somptueuses descriptions des trésors fabuleux et des splendeurs inouïes qu'il a pu admirer à la Cour du Grand Mogol. La richesse d'un tel décor, déployée aux yeux des Français par un écrivain de talent, ne pouvait manquer de séduire les imaginations avant même que la traduction des Mille et Une Nuits n'eût donné le branle de la vogue de l'Orient romanesque ${ }^{14}$ ». Dans cette perspective, la critique établit une comparaison entre la description d'une fête au palais des Indes par Bernier et celle du palais d'Aladdin par Galland et plaide pour la supériorité de Bernier pour la précision des détails et conclut que les contes ne font que " surenchérir » sur la somptuosité décrite dans les récits de voyage ${ }^{15}$. Il fallait rappeler cette filiation entre le conte oriental et les relations des voyageurs en Orient pour comprendre la possible émulation ressentie par Galland dans son désir de «mieux marquer » les mœurs orientales grâce au conte.

8 Il faut encore ajouter que les études historiques sur l'Orient sont de plus en plus précises au dix-huitième siècle, les textes de Mahomet et Confucius sont étudiés et commentés, le Coran est traduit. Les journaux donnent régulièrement des nouvelles des relations diplomatiques entre les pays occidentaux et orientaux. En 1697, La Bibliothèque orientale publiée par Barthélemy d'Herbelot avec l'aide d'Antoine Galland est l'œuvre de référence pour qui veut se renseigner sur l'orient et on comprend que cette somme sera pillée par les auteurs, non orientalistes, désireux de participer à la vogue du conte oriental. Colbert encourage enfin les études de langues orientales; il crée des écoles de "jeunes de langues" (traducteurs) et pousse ces derniers à partir en Orient pratiquer ces langues. Antoine Galland et Pétis de La Croix, tous deux «jeunes de langue » ont profité de cette opportunité. Pétis de La Croix voyage par exemple en Orient pendant dix ans de 1670 à 1680, il passe trois ans à Alep en Syrie puis se rend à Bagdad, puis en Perse (Basra, Chiraz), à Ispahan, à Constantinople. De 1680 à 1690, il est Secrétaireinterprète de la Marine et traducteur au service de la diplomatie. En 1692, onze ans avant Galland, il obtient une chaire d'arabe au collège royal et en 1695, il devient secrétaire-interprète du roi en arabe, turc et persan. Nous sommes, avec ces hommes, aux sources de l'orientalisme en tant que science qui ne se développera vraiment que dans les années 1770 avec des savants comme Anquetil du Perron.

Avant même que le conte oriental ne rencontre ses premiers succès, on constate qu'une double fonction est accordée aux récits qui concernent l'Orient. Il s'agit de plaire à l'imagination grâce à des relations "exotiques " qui font la part belle aux anecdotes « curieuses » et aux descriptions somptueuses. Il s'agit aussi par l'étude scientifique et politique d'une contrée foncièrement "différente » de participer au progrès du savoir et de la réflexion philosophique. Ces deux mouvements peuvent paraître contradictoires : alors que les savants se fondent sur un principe de "différenciation » et s'efforcent de corriger les préjugés des lecteurs; les écrivains procèdent davantage par des généralisations, tout en suivant l'évolution du savoir, et représentent tour à tour un Orient turc, arabe ou chinois. Cette dialectique se retrouve au cœur du conte 
oriental qui, à ses débuts en tout cas, voudrait associer fiction merveilleuse et érudition.

Les notes dans Les Mille et Une Nuits et Les Mille et Un Jours

$10 \mathrm{Au} v \mathrm{vu}$ de cet horizon d'attente oriental, programmé par les relations des voyageurs comme par les études scientifiques et linguistiques, l'originalité des Mille et Une Nuits peut s'expliquer par une synthèse d'aspirations déjà existantes. Selon Marie-Louise Dufrénoy Les Mille et Une Nuits réalisent «la combinaison intime de deux tendances, exotique et fantastique ${ }^{16}$ ", présentes dans les récits des voyageurs et dans les contes de fées traditionnels. Il convient néanmoins d'ajouter à cette double caractérisation thématique, l'importance d'un art de conter qui va lui aussi être attaché au genre naissant du «conte oriental »: la présence d'un récit cadre, le jeu du suspens, la composition des péripéties en sont quelques caractéristiques. C'est en tout cas à partir de ces deux postulats que vont se multiplier les imitations des contes arabes, créant alors le phénomène de mode. Avant même la publication, à titre posthume, du dernier volume des Mille et Une Nuits en 1717, on lit Les Aventures d'Abdalla, fils d'Hanif, de l'abbé Bignon (1712), Gueullette publie Les Mille et Un Quart d'Heures, contes tartares (1715) et Les Mille et Une Soirées (1732). Les publications se succèdent à un rythme soutenu jusque dans les années 1750 .

11 La note de bas de page, informative et/ou explicative, est un signe visible du premier postulat : l'alliance de la fiction et de l'érudition. Mais son statut particulier, étudié par Genette, permet de mettre en question la finalité d'une telle alliance et les conditions de sa réalisation. La note de bas de page appartient au paratexte. Le lecteur a donc le choix de lire ou de ne pas lire ces notes : « [...] de lecture facultative elles ne s'adressent par conséquent qu'à certains lecteurs : ceux qu'intéressera telle ou telle considération complémentaire ou digressive dont le caractère accessoire justifie précisément le rejet en note ${ }^{17}$ ». Deux niveaux de lecture sont alors potentiellement sollicités. A priori, l'auteur sollicite une lecture informative qui profiterait de la note pour enrichir le savoir sur l'Orient offert par le corps du conte. Ce type de lecture rejaillit sur le genre même du conte oriental considéré comme fiction « documentaire ». Cependant, parce qu'elle est facultative et "accessoire " pour reprendre le terme de Genette et parce qu'elle est particulièrement visible, elle peut redoubler le texte. Pétis de La Croix le dit d'ailleurs explicitement dans sa préface, les notes sont un «surplus » par rapport aux informations données par le corps du texte :

[...] ces contes sont aussi utiles qu'agréables; en effet, on y marque exactement la géographie; on y peint les mœurs et les coutumes de différents peuples de l'Asie. [...] Les mets, les boissons les habillements, tout caractérise les nations dont on y parle. Outre cela, toute la morale des musulmans y est répandue. On y apprend une partie de leur théologie. De plus, le traducteur y a joint une infinité de remarques curieuses $[. . .]^{18}$.

12 On comprend que la nature de la note de bas de page autorise des détournements et Gueullette y est particulièrement sensible dès 1715 . Gérard Genette décrit l'intrusion de notes dans une fiction comme "un coup de pistolet référentiel dans le concert fictionnel ${ }^{19}$ ». La note provoque en effet une rupture dans le régime énonciatif, elle peut donc laisser entendre la voix de l'auteur. En instaurant un second niveau de discours, la note permet un jeu de distance par rapport au système énonciatif du texte lui-même. Sans même aller jusqu'à la parodie, le choix de la note de bas de page se révèle problématique : c'est afficher une vocation scientifique tout en la remettant en cause en la reléguant dans les marges du texte. 
13 Avant la publication du premier tome des Mille et Un Jours en 1710, huit volumes des Mille et Une Nuits ont déjà paru, soit dans la collection Garnier Flammarion deux tomes et demi, 900 pages. On trouve au total une cinquantaine de notes, soit en moyenne une note toutes les dix-huit pages. On ne trouve aucune note jusqu'à la quatre-vingtième nuit, c'est-à-dire les voyages de Sindbad. Commence alors une série de notes géographiques, par exemple : Balsora : "port de mer sur le Golfe persique », les îles de Vakvak "à quatre mille cinq cents lieues de l'Abyssinie »: "Ces îles, selon les Arabes, sont au-delà de la Chine et ainsi appelées d'un arbre qui porte un fruit de ce nom. Ce sont sans doute les îles du Japon, qui ne sont pourtant pas si éloignées de l'Abyssinie ${ }^{20}$ ». Cette note est caractéristique du statut du traducteur-conteur qu'est Galland: l'information est de source sûre mais le savant intervient en faisant part de ses doutes (" pourtant »). Dans ces huit tomes, on trouve au total neuf notes géographiques (18\%), quatre notes historiques, concernant surtout les grandes familles de califes (8\%), sept concernent la religion et les croyances (14\%), dix ont rapport aux mœurs, coutumes et vie quotidienne $(20 \%)$ et la majorité des notes sont des traductions de mots orientaux (40\%). Le rôle documentaire est donc primordial, chaque intervention se justifie, par exemple, pour une note que l'on pourrait croire gratuite, Galland précise : «Tous les Orientaux couchent en caleçon et cette circonstance est nécessaire pour la suite ${ }^{21} »$. Dans certaines notes, le commentateur prend tout de même le relais du conteur. Par exemple, lorsque Sindbad se bat contre un géant, on lit :

Après qu'il eut achevé son détestable souper, il se coucha sur le dos et s'endormit.

D'abord que nous l'entendîmes ronfler selon sa coutume, neuf des plus hardis

d'entre nous et moi, nous prîmes chacun une broche, nous en mîmes la pointe dans

le feu pour la faire rougir et ensuite nous la lui enfonçâmes dans l'œil en même

temps et nous le lui crevâmes ${ }^{22}$.

La note de ce passage est la suivante : «Il est à croire que l'auteur arabe a tiré ce conte de l'Odyssée d'Homère ». Galland rappelle ici que ses contes sont authentiquement arabes et sollicite en même temps la culture de son lectorat en référant, en quelque sorte, au premier de tous les conteurs. Deux autres notes sont d'ailleurs du même ordre, deux épisodes de l'histoire des amours de Camaralzaman sont rapprochés de l'histoire médiévale de Pierre de Provence et la belle Maguelonne ${ }^{23}$. Ces remarques ne concernent pas la civilisation arabe mais s'adressent à un public lettré, tout en tissant, discrètement, des liens entre le fonds oriental et le fonds occidental, comme une nouvelle translatio studii.

Dans Les Mille et Un Jours, les notes sont plus nombreuses et plus variées que dans Les Mille et Une Nuits. On trouve cent vingt-quatre notes au total : soit une note toutes les deux pages et demi dans un volume moins important que celui de Galland (481 pages dans l'édition Sebag). Le changement est aussi qualitatif: si les traductions restent importantes (16\%), les notes géographiques sont très peu nombreuses $(4 \%)$. Ce qui ne signifie pas que l'intérêt pour la situation des contes soit moindre, le corps du texte fait une large place aux voyages des héros, précisant mers, fleuves et territoires traversés. Pétis de La Croix s'attache encore plus que Galland à la vie quotidienne et à la vie de la cité. Après la curiosité pour les mœurs orientales, Pétis de La Croix introduit des " curiosités » artistiques, esthétiques ou culinaires : les tissus, la poésie, les comédiens, la porcelaine, les palanquins suscitent des explications. Les notes consacrées à la cuisine et à la musique sont particulièrement représentatives de cette tendance ${ }^{24}$ et étaient absentes des Mille et Une Nuits. En outre, l'orientaliste donne à son public, mondain, des éléments de comparaison avec les habitudes européennes, par exemple à 
l'occasion de cette note sur le vin de riz : «Le vin de riz est de couleur d'ambre et aussi délicat que le vin d'Espagne » (p. 199). À l'occasion de réjouissances à Moussel, on lit : "Les boutiques des asouaques et des badistans, furent tapissées en dedans et en dehors... »; une première note explique le premier terme : ce sont des rues marchandes et une seconde le deuxième terme : «c'est un lieu comme la Foire Saint Germain ou le Palais, tout rempli de boutiques de bijoutiers» (p. 161) ou encore lors d'un spectacle chez le roi de la Chine : «Les comédiens chinois tant ceux du roi que les autres jouent sur le champ tout ce qu'on leur ordonne de jouer, comme les comédiens italiens " (p. 199). Comme pour Les Mille et Une Nuits, les notes des Mille et Un Jours ne sont pas des " pillages » de La Bibliothèque orientale et sont donc probablement dues à leur auteur, à son savoir personnel sur l'Orient. C'est peut-être pourquoi les notes sont, en majorité, laconiques. Par exemple, pour le terme aga, nous lisons dans le recueil «chef des eunuques » (p. 162). Dans La Bibliothèque orientale, l'article est beaucoup plus développé : c'est un commandant mais chez les Turcs, l'« aga des janissaires » est un colonel, enfin on apprend que ce nom est "donné aux eunuques car ils ont tous les commandements ${ }^{25}$ ». La fonction première de ces notes est donc documentaire et informative, elles répondent à une demande de "sérieux " ainsi que le précise Pétis de La Croix : «[...] pour sauver s'il se peut cet ouvrage du mépris de certains lecteurs sérieux qui ne sauraient souffrir les fictions les plus ingénieuses et à qui le plus agréable livre ne saurait plaire si le crédit de la vérité ne le rend recommandable ${ }^{26}$ ".

Les notes "linguistiques ", c'est-à-dire les traductions de mots orientaux en français sont intéressantes pour approcher le projet du conte oriental à ses débuts. Toute traduction pose en effet la question de la propriété du langage, de son aptitude à rendre les mots d'une civilisation à une autre, d'où la préférence parfois, dans le corps du texte, du mot original plutôt que de son interprétation en français : le Rommanaschi est sans doute sans équivalent en Europe, c'est pourquoi le traducteur garde le terme indien et donne une explication brève en note : " c'est un mets où il entre des grains de grenade » (p. 89). Mais, ces mots non traduits ont aussi une fonction "exotique ", ils sont le pittoresque linguistique du recueil. Enfin, l'interprétation des noms propres peut être utile dans une perspective dramatique, si l'on pense au lien existant entre le nom et le caractère ou la destinée de la personne auquel le lecteur de contes traditionnels est habitué, par exemple la nourrice porte le nom approprié de Sutlumemé ("gorge de lait», p. 40), l'esclave Adelmuk qui se suicide en vertu de son origine royale : « équité du royaume » (p. 208), et le bon vizir Atalmuk : " présent fait au royaume» (p. 214). Les notes répondraient donc à un principe de concordance, d'explicitation et d'élucidation. Cependant, le changement de régime énonciatif qui les définit pourrait bien laisser place à quelque discordance.

Certaines notes inversent, en effet, le processus de traduction qui va de la langue étrangère vers le français pour donner en note la traduction orientale de l'expression ou du mot français. Par exemple, luth est annoté Aoud (p.112), « un potage de jus de mouton »: "Asche rischté y guipa» (p. 123) et pour les escarpins des baladins chinois : «on les appelle naleines» (p.199). L'intérêt de telles notes est plus problématique: elles n'ont pas une fonction d'élucidation mais révèlent une érudition gratuite. La plus caractéristique est la suivante : "Je vous conjure par le grand prophète notre dieu... », dit le gouverneur d'Hormoz et on lit en note: Allah (p. 297) alors que l'on s'attendrait à ce qu'elle explicite le nom du prophète désigné. Tous les lecteurs savent que le Dieu des musulmans se nomme Allah, ils l'ont déjà rencontré à maintes reprises dans Les Mille et Une Nuits, les relations de voyageurs, mais aussi Les Mille et Un jours. La note se désigne 
elle-même et signale au lecteur la naissance d'une convention. En outre, la note semble parfois appeler le texte et non l'inverse. On se souvient de l'exemple de Galland qui justifiait l'intrusion d'une note curieuse par la suite des événements, plus de scrupule chez Pétis de La Croix : une simple interjection déclenche un long exposé didactique. Le cadi face à la beauté de Zemroude s'écrie: «Par le sacrifice du mont Arafate, [...] je n'aperçois en vous aucun défaut... », la note s'attache au terme Arafate:

C'est une montagne voisine de La Mecque : les Mahométans croient qu'Adam et Ève ayant été chassés du paradis l'un vers l'Orient, l'autre vers l'Occident, à cause de leur désobéissance, ils errèrent sur la terre pendant cent vingt ans par pénitence en se cherchant ; et qu'enfin ils se rencontrèrent et se reconnurent sur le mont Arafate qui pour cette raison a tiré son nom du mot arabe arafa qui signifie reconnaître. Le dixième jour de la lune de $\mathrm{Zu} \mathrm{l-haja,} \mathrm{qui} \mathrm{est} \mathrm{la} \mathrm{dernière} \mathrm{des} \mathrm{douze} \mathrm{de} \mathrm{l'année}$ arabique, jour appelé Aïd al-adha, c'est-à-dire fête du sacrifice, les pèlerins de la Mecque y font une procession générale nommée Tavof $[. . .]^{27}$.

Suit la description longue et détaillée de cette procession et du sacrifice qui la termine. Il y a donc un double décalage : l'interjection à fonction phatique entraîne une note érudite à fonction informative. D'autre part, la note crée un effet de décontextualisation - une scène de séduction entraîne un exposé religieux -, qui pose la question d'une possible lecture parodique. On peut en outre se demander qui va lire cette note, qui interrompt le fil d'une scène comique de tromperie.

Les notes récurrentes ajoutent encore un niveau de lecture possible. Les houris sont expliquées à trois reprises avec toujours plus de précisions: "ce sont les filles du paradis de Mahomet» (p.151), «Filles du paradis de Mahomet qui ne vieillissent jamais » (p. 155), «Les houris, comme on l'a déjà dit dans les jours précédents, sont les filles du paradis de Mahomet. Par un miracle de l'Alcoran, elles n'ont jamais que quinze ans et sont toujours neuves quoiqu'elles fassent le bonheur des bienheureux musulmans» (p. 276). C'est un élément de civilisation qui ne peut que frapper les imaginations occidentales, les récits de voyageurs ne manquent d'ailleurs pas d'y référer. On remarque alors un certain suspens dans la gradation ménagée d'une note à l'autre, qui joue sur les attentes du lectorat. En outre, la récurrence sollicite la mémoire, vertu essentielle à tout lecteur de conte et à tout conteur. Les renvois de note à note sollicitent, dans cette perspective, une lecture transversale du recueil qui n'est pas habituelle. Nous avons également trois occurrences de la Table fatale dans le corps du texte, une première note très développée explique l'origine et la signification de cette expression (Jour 39, p. 126). Les deux notes suivantes renvoient à la première (p. 139 : «Voyez Jour XxxIX et note» et p. 277 : «Voir plus haut, jour XxxIx et note»). Une note à propos de l'île de Sérendib renvoie à la longue note sur le Mont Arafate citée ci-dessus :

[...] C'est sur une montagne de cette île que plusieurs auteurs orientaux prétendent qu'Adam et Eve se rencontrèrent lorsqu'ils eurent fait le tour du monde. Cependant, d'autres auteurs mahométans prétendent que cette rencontre se fit sur le mont Arafate auprès de la Mecque, voir plus haut, Jour LII, note ${ }^{28}$.

L'annexe érudite est tout aussi fortuite que celle du mont Arafate : une indication de lieu : «Je suis la fille du roi de Sérendib » entraîne un exposé religieux. Le recueil de contes autoriserait alors une lecture anthologique : on peut choisir les contes ou leur ordre de lecture. C'est pourquoi, il peut être nécessaire d'expliquer plusieurs fois des principes de civilisation importants ou étonnants, la Table fatale en est un, l'existence de houris, un autre. En tout cas, que l'on interprète ces décalages par rapport à ce que l'on attendrait des notes, comme un jeu avec les conventions, privilégiant l'aspect 
facultatif de la lecture, ou comme une conscience du genre particulier du recueil, ces notes et leur traitement sollicitent une participation du lecteur.

Fortune des notes dans le conte oriental

Le recueil des Mille et Un Jours fondé sur des manuscrits originaux et des notes prises par Pétis de La Croix repose sur une connaissance authentique de l'Orient et est le premier à intégrer des histoires religieuses (le conte d'Aboulfaouaris par exemple), mais il contient aussi en germe les virtualités ludiques et parodiques de la dialectique entre savoir et fiction à l'œuvre dans le conte oriental. Notre propos n'est pas de dresser un inventaire des usages de la note après Galland et Pétis de La Croix mais à l'aide de deux exemples, Gueullette et Caylus, de montrer comment sera exploité le « jeu » possible entre la note et le texte entrevu dans Les Mille et Un Jours. Gueullette privilégie la voie parodique, Caylus choisit de revenir à un usage « sérieux » de la note.

Gueullette use des notes avec la plus grande variété : des notes sérieuses, ludiques, gratuites ou nécessaires se côtoient dans ses ouvrages, le tout dans une visée globalement parodique. Il se glisse explicitement dans le sillage de Galland et de Pétis de La Croix et entend profiter de l'enthousiasme suscité par les premiers contes orientaux. Les Mille et Un Quart d'Heures, contes tartares, paraissent en 1715, trois ans après le dernier volume des Mille et Un Jours et pendant la publication des Mille et Une Nuits. On y trouve des notes gratuites, répétant le texte, par exemple pour la phrase : «Lorsque Gulguli Chemané entra dans Nanquin, capitale de la Chine... »; Nanquin est annoté ainsi : "Nanquin est une des principales villes de la Chine où il est très certain que Fantru a régné ${ }^{29}$ ». Gueullette reprend également des notes des Mille et Une Nuits ou des Mille et Un Jours. On retrouve, par exemple, la note de Galland sur les caleçons en ces termes : «Dans tout l'Orient, les hommes et les femmes couchent avec des caleçons ${ }^{30}$ ". Cependant, aucune justification diégétique n'apparaît, Gueullette donne le détail pour lui-même. Les longs exposés religieux sont tout aussi fortuits que dans le recueil de Pétis de La Croix. Ainsi, pour le syntagme : «Oui, je jure par les six gouttes de sueur de Mahomet... ", une note explique que Mahomet au paradis, sua de honte sous le regard de Dieu: «il en fit tomber six gouttes hors du paradis, l'une desquelles fit naître sur le champ les roses et le ris ${ }^{31}$ ». À propos des comédiens en Inde, on retrouve la même comparaison que chez Pétis : "Les baladins et les comédiens sont fort communs en Inde; ils jouent avec beaucoup d'esprit et la plupart du temps sans préparation à peu près comme faisaient en France nos comédiens italiens ${ }^{32}$ ». Gueullette connaît par ailleurs très bien ce théâtre, à propos duquel il a écrit des Notes et souvenirs.

La filiation avec Galland et Pétis de La Croix est explicitement avouée quelques années plus tard dans l'«Avis au lecteur» des Sultanes de Guzarate ou les songes des hommes éveillés (aussi baptisé Les Mille et Une Soirées) en 1732: «Messieurs Galland et Pétis de La Croix, ou du moins ceux qui leur ont prêté leur plume pour rédiger et écrire les contes arabes, persans et turcs, paroissent avoir épuisé la matière et il semble qu'il n'y ait plus à glaner après eux ». La tonalité est d'emblée parodique. Gueullette n'hésite pas à retranscrire les rumeurs au sujet de la paternité des contes dits de Pétis de La Croix ${ }^{33}$ mais insinue des soupçons pour Galland également. Il reprend le topos de La Bruyère : " Tout est dit et l'on vient trop tard depuis plus de sept mille ans qu'il y a des hommes et qui pensent [...], l'on ne fait que glaner après les Anciens et les habiles d'entre les modernes $^{34}$ ». Galland et Pétis de La Croix sont les Anciens du conte oriental, auxquels il faut rendre hommage, avant de s'en distancier. Après cette ouverture, vient une justification des notes particulièrement abondantes dans ce nouveau recueil : 
Il me reste à dire que ce n'est pas sans raisons que j'ai mis à ces avantures des notes géographiques et historiques un peu plus amples que l'on a coutume de le faire en pareil cas, outre qu'il y a nécessairement dans ces sortes d'ouvrages des endroits qui ont besoin d'explication, surtout pour les Dames ; $j$ 'ai cru devoir les appuyer et principalement ceux qui regardent l'histoire fabuleuse, de l'autorité de la bibliothèque orientale, ou des plus célèbres voyageurs qui ont parcouru ces vastes pays, persuadé que le lecteur ne me saura pas mauvais gré de ces remarques, qu'il sentira bien m'avoir coûté beaucoup de tems et de lecture. reprises, des "recopiages" de La Bibliothèque orientale et de quelques relations de voyages (Thévenot en particulier). Gueullette n'est pas le seul à pratiquer cette technique du copier-coller avant l'heure. Régine Jomand-Baudry a montré que JeanFrançois Melon ne procède pas autrement dans son unique roman Mahmoud le Gasnévide: La Bibliothèque orientale, les récits de voyage de Paul Lucas et de Chardin, les historiens sont sollicités pour enrichir les notes, dans « un souci de transmettre une image juste de l'Orient ${ }^{35}$ ». Le projet de Gueullette n'est donc pas le même que celui de Pétis de La Croix ou de Galland qui pour les notes, utilisaient leur propre savoir. L'intention parodique perce sous cette longue justification, qui aboutit à l'ennui, ennui de l'auteur (« beaucoup de tems et de lecture »), qui provoquera fatalement l'ennui du lecteur. Gueullette se plie donc à une convention qu'il croit obligatoire plus qu'à un zèle pédagogique. Quelques exemples de notes pourront le confirmer. On trouve une longue note sur les houris (bien sûr), mais le paratexte prend vite le pas sur le texte même : en deux pages on nous explique outre ce que sont les houris, toutes les lois du paradis avec force détails pour achever ainsi : "Tant d'extravagances et de puérilités avec des détails aussi ridicules sont rapportés dans Le Second Volume de la religion des Turcs par Echialle Mufti, chapitre 48-49 $9^{36}$ ». La source même est ridiculisée, c'est dire la vanité du projet didactique ${ }^{37}$. Gueullette n'hésite pas non plus à renvoyer à ses propres œuvres, par exemple à ses Contes chinois de $1723^{38}$. Plus loin, c'est la note qui semble appeler le texte, dans une logique accumulative qui finit par noyer le sens même de la phrase concernée : en huit lignes de texte original, on compte dix renvois à des notes : Bahalul décide de faire un pèlerinage à La Mecque (1) :

Pour cet effet, il partit d'Ormuz et après un assez long trajet, étant parvenu par la mer rouge jusqu'à Gidda (2), il se rendit par terre à la Mecque en trois jours; là après avoir fait sept fois le tour de l'Oratoire (3), y avoir baisé la pierre (4) noire, vu la Goutière (5) d'or, fait sa station et le Corban (6) sur le mont Arafat, bu de l'eau du puits ZemZem (7), fait sept autres tours entre Safa (8) et Merve et jeté des pierres dans la vallée de Menah (9). Mon mari prit la route de Médine (10)... ${ }^{39}$

L'intention est ludique, Gueulette joue ostensiblement avec les notes, devenues conventions du genre «conte oriental » et accentue à cet effet la distanciation déjà sensible dans Les Mille et Un Jours arrivant après Les Mille et Une Nuits.

En revanche, alors que l'on pensait la mode des contes passée, en 1743, les Nouveaux contes orientaux du Comte de Caylus renouent avec l'usage érudit de la note de bas de page dans le conte oriental. Ces contes sont "nouveaux", car inédits et surtout authentiques. Le Comte utilise des sources orientales écrites originales, il entend faire œuvre de savant et non d'imitateur opportuniste: "Je compte beaucoup sur la nouveauté de celui-ci : je pourrais même, sans blesser la modestie, en faire un plus grand éloge n'ayant point d'autre part à ce recueil que celui de l'avoir rassemblé ${ }^{40}$ ». Ces origines authentiques fondent un recueil sérieux : un tiers des textes (« Histoire de la naissance de Mahomet », «Histoire d'un derviche», «Jugement de Dgerberi » par 
exemple) reprend l'hagiographie musulmane, par exemple «l'histoire de Dakianos et des sept dormans " suit parfois textuellement le Coran ${ }^{41}$. Ce recueil, à la différence de celui de Gueullette, mais à l'image de ceux de Galland et Pétis de La Croix, se fonde sur un véritable savoir oriental. Le comte de Caylus a consulté de multiples ouvrages, a voyagé au Moyen-Orient (en 1716) et avait de nombreux contacts avec les milieux orientalistes. Raymonde Robert s'est intéressée à la personnalité de cet homme qui fréquente le salon de $\mathrm{M}^{\mathrm{lle}}$ Quinault, se mêle aux fêtes du bas-peuple et est aussi membre honoraire de l'Académie des Inscriptions et des Belles lettres en 1742. Elle ajoute que ces contes «ne sauraient être confondus avec la masse insipide des contes que la vogue a produits ${ }^{42} »$. On y trouve certes une part de jeu, par exemple dans le conte cadre qui joue avec les «lois» du genre: le roi a une insomnie, la jeune fille du geôlier doit parvenir à l'endormir grâce à des contes. Cependant, au sein même du recueil on ne trouve " aucun indice [qui] suggère une interprétation ironique ou dépréciative ${ }^{43}$ ». Il en est de même pour les notes, elles sont moins nombreuses que dans les recueils de Gueullette et il n'y a pas de notes ludiques. Par exemple, on trouve une longue note à l'occasion d'une expression phatique, méthode ironique pratiquée par Pétis et Gueullette, mais ici c'est bien l'expression, authentique, qui est expliquée, elle n'est pas seulement un prétexte à l'érudition. On lit dans le texte : "Il serait plus aisé de vous expliquer l'histoire de l'oiseau Anka que de vous persuader de mes malheurs ", la note donne une description de l'oiseau et s'achève ainsi : «[...] Les Orientaux disent que depuis longtemps cet oiseau s'est retiré sur la montagne de Kaf qui entoure le monde et que cet endroit est inconnu. C'est ce qui les engage à dire: "Autant vaudroit-il vous enseigner la demeure des Anka, ou vous donner de ses nouvelles" 44 ». Le comte inclut même des documents inédits au cœur de son recueil, venant interrompre la fiction. Il s'intéresse, par exemple, à la signification symbolique des présents que se font les Orientaux. Une note de l'«Histoire de Naerdan et Guzulbec » explique qu'un petit paquet de gingembre signifie : « mon cœur ne brûle que pour toi », un petit morceau de drap bleu: «Je suis toujours amoureux de vous ${ }^{45}$ ». Deux histoires plus loin, l'auteur intervient pour revenir sur cet usage et insère une liste des présents et de leur signification :

L'on a cru pour la plus grande intelligence d'un passage de l'histoire du roi de Kachemir, et pour satisfaire la curiosité du lecteur sur un usage de l'orient, devoir lui donner une liste de quelques mané ou salams qui sont des présens simples, en même tems que des signes dont les amans conviennent pour tromper la vigilance de ceux qui s'opposent à leur bonheur ${ }^{46}$.

27 La première phrase revient, en quelque sorte, aux sources du genre du conte oriental puisqu'elle justifie la note, et l'annexe documentaire, par la compréhension de la fiction, ce que faisait Galland, et par le recours à la " curiosité ", dont nous avons fait le mot clé de la réception « orientalisante » au début du dix-huitième siècle.

Dès son apparition, le conte oriental a donc recours à la note de bas de page qui, au cours de son histoire, deviendra une sorte de loi du genre. Le recours à un signe caractéristique des ouvrages scientifiques n'est pas gratuit mais renvoie à la nature hybride de ce genre naissant. Galland et Pétis de La Croix abordent le conte avec le sérieux et la rigueur du linguiste et du savant. Pourtant, la note de bas de page est visiblement un "surplus » par rapport au texte du conte déjà riche d'enseignements sur les «mœurs et coutumes » orientales. De lecture facultative, elle peut devenir le lieu du commentaire critique mais aussi des prises de distance ludiques par rapport à la civilisation orientale ou par rapport au genre du conte. Incidemment, dans les marges 
du texte, se joue peut-être alors le destin du conte oriental qui, après Galland et Pétis, va emprunter deux voies, qui se croisent parfois, celle de la parodie et celle de la philosophie.

\section{NOTES}

1. Les Mille et Une Nuits, «Avertissement », Garnier Flammarion, 1965, p. 21. Nous soulignons.

2. La seule note de ce conte parodique à décor oriental se reporte en effet au nom et à l'œuvre d'un « savant chinois » : "Cham-hi-hon-chu-kah-hul-chi, Hist. Litt. de la Chine. Pékin, 1306, p. 155, I vol. », dans Claude Crébillon, Euvres complètes, Garnier, 1999, p. 270. Cependant, Crébillon se moque ici d'une pratique qui sera celle des " profiteurs » de la vogue du conte oriental, adeptes de la note, on le verra avec Gueullette, mais ayant besoin de documentation externe pour les enrichir, ce qui n'est pas le cas de Galland et Pétis de La Croix qui détiennent personnellement ce savoir. 3. Le numéro spécial de la Revue dix-huitième siècle consacré à l'Orient pose ce problème de délimitations dans l'introduction. Les articles d'érudits et d'Orientalistes tâchent d'y répondre par des critères géographiques, historiques, mais aussi religieux, Revue dixhuitième siècle, $\mathrm{n}^{\circ}$ 28, 1996.

4. Mohamed Abdel-Halim, Antoine Galland, sa vie et son œuvre, Nizet, 1964, p. 149.

5. Ce sont des tribus mongoles d'Asie centrale.

6. Antoine Galland, Paroles remarquables des Orientaux, 1694, cité par Pierre Martino, L'Orient dans la littérature française du dix-septième et dix-huitième siècle, Hachette, 1906. 7.R. Etiemble, L'Orient philosophique au dix-huitième siècle, CDU, 1956, p. 38.

8. «La visite de Suleiman Aga avait mis l'Orient à la mode, les mœurs de ses habitants, leur langage fleuri, leur vanité imperturbable et naïve faisaient le sujet ordinaire des conversations. ", L. Vandal, « Molière et le cérémonial turc à la cour de Louis XIV », Revue d'histoire diplomatique, 1888, cité par H. Desmet-Grégoire, Le Divan magique, l'Orient turc en France au XVIIIe siècle, L'Harmattan, 1994, p. 19.

9. Par exemple en 1714, l'ambassadeur persan Mehemet Reza Beg ayant été perçu comme un « homme cruel et excentrique », le préjugé s'est ensuite appliqué à tous les Musulmans ; en revanche en 1721, le Turc Mehemet Effendi fit bonne impression en se montrant très réceptif à la société française et déclencha un mouvement de sympathie. 10. Cette représentation se diffuse par l'intermédiaire des Lettres édifiantes et curieuses écrites des missions étrangères par quelques missionnaires de la Compagnie de Jésus, de 1702 à 1776.

11. P. Martino, ouvr. cité, p. 51.

12. Cité par P. Martino, Ibid., p. 63.

13. Ibid., p. 70.

14. M.-L. Dufrénoy, L'Orient romanesque en France (1704-1789), tome III, Rodopi, Amsterdam, 1975, p. 19.

15. Ibid., p. 98-100.

16. Ibid., p. 100. 
17.G. Genette, Seuils, éditions du Seuil, 1987, p. 297.

18. Les Mille et Un Jours, " Avertissement », éd. Paul Sebag, C. Bourgois éditeur, 1981, p. 34. Nous soulignons.

19.G. Genette, ouvr. cité., p. 307.

20. Les Mille et Une Nuits, éd. citée, t. I, p. 232-233.

21. Ibid., p. 324.

22. Ibid., p. 250.

23. Les Mille et Une Nuits, éd. citée, t. II, p. 190 et p. 203.

24. Voir par exemple pour la musique p. 162 (tambouras et deffs), p. 302 (tambour, tarzana, musicien Abdelmoumen), p. 304 (Zils), p. 306 (le mode musical Uzzal) et pour la cuisine qui représente $10,5 \%$ des notes : le fiquaa (p. 58, 287, 437), le rommanaschi (p. 89), les concombres de Perse (p. 222) la chourva et le tziberica (p. 144) etc.

25.B. d'Herbelot, La Bibliothèque orientale ou Dictionnaire universel contenant généralement tout ce qui regarde la connaissance des peuples de l'Orient, Compagnie des Libraires, 1697, p. 41.

26. Avertissement des Mille et Un Jours, p. 35.

27. Les Mille et Un Jours, p. 154.

28. Ibid., p. 261.

29. Gueullette, Les Mille et Un Quart d'Heures, J. B Mazuel, 1715, p. 69.

30. Ibid., p. 205

31. Ibid., p. 221.

32. Ibid., p. 305.

33. Les contes persans sont Les Mille et Un Jours, les contes turcs : La Sultane de Perse et les quarante vizirs.

34. La Bruyère, Caractères, section « Des ouvrages de l'esprit », éd. E. Bury, Le Livre de Poche, p. 124.

35. Elle montre ensuite comment la note est intégrée dans un subtil système de déchiffrement allégorique, l'Orient de Melon renvoyant à l'Europe des années 1720 . R. Jomand-Baudry, " Signification des notes auctoriales dans la fiction orientale du début des années 1730 », Le Roman des années trente, études réunies par A. Rivara, PU Saint-Étienne, 1998, p. 133.

36. Gueullette, Les Sultanes de Guzarate, Pierre Prault, 1732, p. 68-69.

37. Dans son article, Régine Jomand-Baudry explique ces interventions critiques de Gueullette par sa double vocation de traducteur et de commentateur et les rattache à la « visée morale » des contes, toujours prégnante chez Gueullette, article cité, p. 140-141.

38. Gueullette, Les Sultanes de Gurazate, p. 87.

39. Ibid., p. 171-172.

40. "Dédicace à Madame*** ", Nouveaux contes orientaux, dans Nouveau cabinet des fées, t. XI, Slatkine Reprints, Genève, 1978.

41.R. Robert, « Le comte de Caylus et l'Orient : la littérature aux prises avec le même et l'autre », SVEC, $\mathrm{n}^{\circ} 154,1976$, p. 1838.

42. Ibid., p. 1825.

43. Ibid., p. 1838.

44. Caylus, Nouveaux contes orientaux, éd. citée, p. 282.

45. Ibid., p. 135.

46. Ibid., p. 181. La liste se trouve aux pages 182-183. 


\section{RÉSUMÉS}

La présence de notes de bas de page dans les premiers recueils de contes orientaux, ceux de Galland et de Pétis de La Croix en particulier, est un signe ostensible du statut hybride du genre naissant qu'est alors le « conte oriental ». La fiction merveilleuse doit en effet s'accommoder d'un savoir sur l'Orient, les deux savants entendant faire partager leur connaissance du monde oriental. Cependant, par son statut marginal et accessoire, la note est aussi le lieu privilégié de la distance critique ou ludique. Les recueils de Gueullette et Caylus nous servent alors d'exemples pour évoquer la double destination de la note du conte oriental, parodique et sérieuse.

The Meaning of Footnotes in the First Oriental Tales. The presence of footnotes in the first collections of Oriental tales, those composed by Galland and Pétis de La Croix in particular, is a clear sign of the hybrid status of the emerging genre. The fantasy in the fiction must make room for an erudite approach of the Orient, both writers being eager to share their knowledge of the Oriental world. However, the marginal and ancillary status of the footnote makes it a favourite spot for the expression of critical distance or playful treatment of the original material. The collections published by Gueulette and Caylus provide us with many illustrations of this double aspect of the footnote in Oriental tales, at the same time serious and parodical.

\section{AUTEUR}

\section{CHRISTELLE BAHIER-PORTE}

Université de Saint-Étienne 